\title{
Henoch Schonlein Purpura Presenting with Intussusception in a 7-Year-Old Female in South-West Nigeria: A Case Report
}

\author{
M. B. Adebola*, M. M. Ogundeyi, O. B. Adeogun, I. O. Oguntade \\ Paediatrics Haematology, Oncology Unit, Federal Medical Centre, Abeokuta, Nigeria \\ Email: *adebola.mb@fmcabeokuta.org
}

How to cite this paper: Adebola, M.B., Ogundeyi, M.M., Adeogun, O.B. and Oguntade, I.O. (2021) Henoch Schonlein Purpura Presenting with Intussusception in a 7Year-Old Female in South-West Nigeria: A Case Report. Open Access Library Journal, 8: e7430.

https://doi.org/10.4236/oalib.1107430

Received: April 16, 2021

Accepted: May 28, 2021

Published: May 31, 2021

Copyright (๑) 2021 by author(s) and Open Access Library Inc.

This work is licensed under the Creative Commons Attribution International License (CC BY 4.0).

http://creativecommons.org/licenses/by/4.0/

(c) (i) Open Access

\begin{abstract}
Henoch-Schonlein Purpura (HSP) is the most common childhood systemic vasculitis. It is characterized by leucocytoclastic vasculitis and IgA deposition in the skin, joints, gastrointestinal tract, and kidneys, and typically manifests with purpura, arthritis/arthralgia, abdominal pain and nephritis. About 70\% of children with HSP develop abdominal pain from submucosal haemorrhage and bowel wall oedema. These may result in gastrointestinal complications such as intussusception and may impact adversely on outcome. We report a case of HSP in a 7-year-old female who had presented with a recurring course of palpable purpura restricted to both lower limbs, painful swelling of the right knee joint, as well as severe abdominal pains which later became associated with passage of bloody stools. She subsequently had an exploratory laparotomy for sonographically confirmed intussusception following which she was referred to the haematology clinic on account of persistence of purpuric limb lesions post-surgery. This report highlights HSP as the commonest childhood systemic vasculitis. Its prognosis in childhood is generally excellent, with a self-limited course in most cases. Gastrointestinal complications such as intussusception, may, however, impact adversely on outcomes, while renal involvement may progress to chronic kidney disease.
\end{abstract}

\section{Subject Areas}

Pediatrics

\section{Keywords}

Henoch-Schonlein Purpura, Vasculitis, Intussusception

\section{Introduction}

Henoch-Schonlein Purpura (HSP) is the most common childhood systemic vascu- 
litis [1] [2]. It is characterized by leucocytoclastic vasculitis and IgA deposition in the skin and joints [2]. HSP occurs worldwide and affects all ethnic groups, but is more common in Caucasians and Asian populations.

The incidence of HSP is estimated at 10-20/100,000 children per year [2] [3], and it affects males more than females, with a $1.2-1.8: 1$ male-female ratio. Approximately $90 \%$ of HSP cases occur in children, usually between the ages of 3 years and 10 years [1] [2]. HSP is distinctly less common in adults, in whom severe and chronic complications are often encountered [3] [4]. HSP is more common in the winter and spring, and is unusual in summer months [3]. Many cases of HSP follow a documented upper respiratory infection [3].

The exact pathogenesis of HSP remains unknown [2]. It occasionally clusters in families, suggesting a genetic component. Given its seasonality, and the frequency of preceding upper respiratory infections, however, infectious triggers such as group A $\beta$-haemolytic Streptococcus, Staphylococcus aureus, Mycoplasma, and Adenovirus have been suggested [5].

The common finding of deposition of IgA, specifically IgA1, suggests that HSP is a disease mediated by IgA and IgA immune complexes [3]. Skin biopsies demonstrate vasculitis of the dermal capillaries and post-capillary venules, while Immunofluorescence identifies IgA deposition in walls of small vessels of all tissues, accompanied to a lesser extent by deposition of C3, fibrin, and IgM [2].

In addition to the typical rash which is a constant feature of the disease [1], about $80 \%$ of children with HSP develop abdominal pain from submucosal haemorrhage and bowel wall oedema which may serve as lead points for intussusception [1] [2]. Intussusception, though rare, is the most common surgical complication of HSP. Ileal perforations, may occur spontaneously or be associated with intussusception. Ultrasound is recommended as the first diagnostic test.

Unlike in adults where disease is usually more severe and associated with poorer outcomes, the overall prognosis in childhood HSP is generally excellent, with a self-limited course in most case [4]. Gastrointestinal complications, like intussusception, may however impact adversely on outcomes, while renal involvement may progress to end-stage renal disease

We report a belatedly diagnosed case of HSP in 7-year-old female patient who had surgery for intussusception to highlight this disorder and its association with potentially serious gastrointestinal complications.

\section{Case Report}

We present a 7-years old female referred to the haematology clinic of our Centre from the paediatric surgery clinic on account of rash involving both lower limbs (Figure 1). The rash were pink in colour, limited to both legs, and had a waxing and waning course since onset about six weeks earlier.

She had previously presented at the orthopaedics clinic on two occasions: the initial presentation was two weeks prior to the onset of the rash on account of painful swelling involving the left knee joint. The joint swelling was initially 


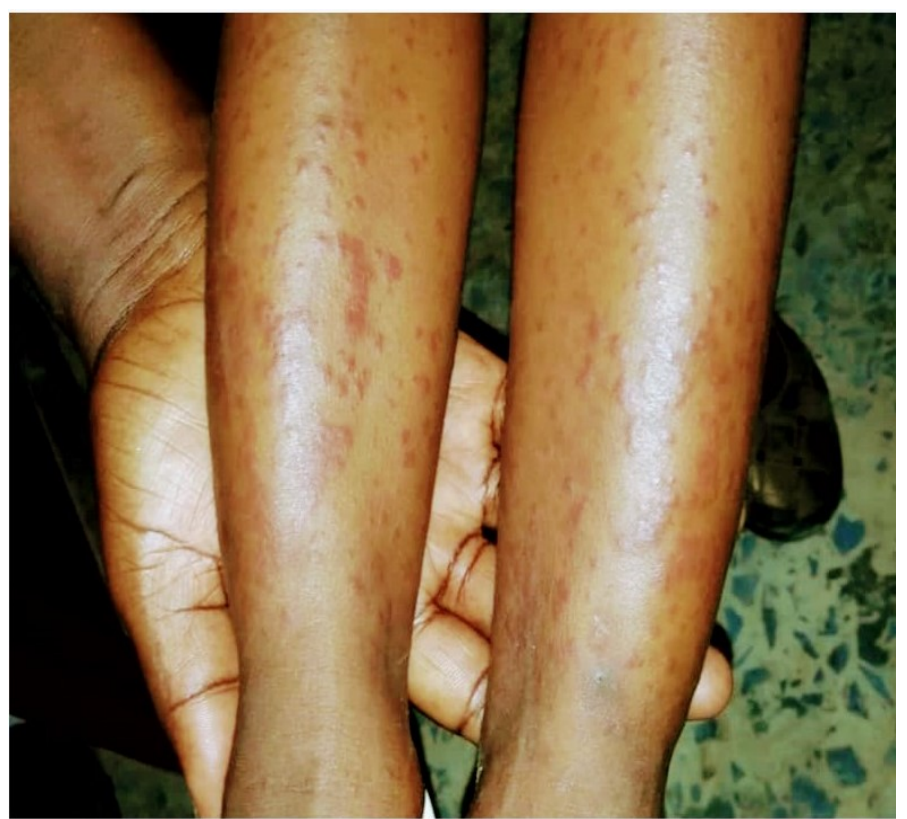

Figure 1. Lower limbs of index patient at presentation showing diffuse palpable purpura bilaterally.

adduced to a fall and was managed with analgesics and a splint applied across the joint. The swelling initially resolved following the aforementioned interventions, only to recur about two weeks later. The second episode of joint swelling was however clearly not related to trauma and was managed as septic arthritis, although X-ray findings were unremarkable. By this time she had developed the bilateral leg eruptions.

She had also been complaining of recurrent episodes of abdominal pains about the same time the initial joint swelling was noticed. The pains were severe, diffuse with no known aggravating nor relieving factors. The abdominal pains had warranted several out-patient visits for which analgesics were administered with no significant resolution of symptoms. About four weeks into illness she presented at the children's emergency room with severe abdominal pains associated, this time with passage of bloody and mucoid stool. A diagnosis of intussusception was considered and subsequently confirmed by ultrasonography. She had an emergency laparotomy performed with resection of a segment of the ileum followed by end to end anastomosis of the edges.

She was subsequently discharged to the surgical out-patient clinic from where she was referred to our clinic on account of the purpuric eruptions which had waxed and waned since onset six weeks earlier.

Examination findings at presentation at our clinic revealed an otherwise healthy school-aged female with palpable purpura limited to both legs. Other findings on physical examination were essentially normal. Her FBC showed leukocytosis and thrombocytosis while ESR was elevated. Bleeding time was within normal limits. Serial E/U/Cr, urinalysis and urine microscopy all remained normal throughout the course of the illness. Serum C3 was ordered, but could 
not be done due to logistic constraints.

Review of intra-operative findings revealed a hemorrhagic, thickened segment of ileum (24 cm long) $30 \mathrm{~cm}$ from ileo-caecal junction, which was resected. Macroscopically, the histopathology report of the resected bowel segment revealed an ileal segment measuring $21 \mathrm{~cm}$ in length, with a hemorrhagic patch in the mid-segment. On microscopy, sections of intestinal tissue showed hyperplastic inflamed mucosa with presence of mononuclear cells and congested vessels. The submucosa showed chronic inflammatory cell infiltrates. No necrosis or ulcers were seen. Her parents were reassured and counseled on the typically self-limiting nature of the disease, Use of corticosteroids was considered, but same was eventually withheld as there was no clear indication as at the time she presented to our clinic. The rash waxed and waned over the next 4 weeks following first contact with our unit (total duration of illness being about 12 weeks), and symptoms have not subsequently reappeared over a one-year follow-up period. Her urinalysis and urine microscopy results have also been normal. She is however still being followed up.

\section{Discussion}

The diagnosis of HSP [2] [6] as revised by the Paediatric Rheumatology European Society is based on the presence of:

Palpable, non-thrombocytopenic purpura (mandatory criterion), in the presence of at least one of:

- Diffuse abdominal pain.

- Any biopsy sample showing predominant IgA deposition.

- Arthritis or acute arthralgia in any joint.

- Renal involvement.

- IgA deposition in any sample in a patient with atypical disease.

The diagnosis of HSP in the present patient was based on the presence of palpable, non-thrombocytopenic purpura limited to both legs, recurrent painful knee swelling, as well as recurrent diffuse abdominal pains with a subsequent sonographic confirmation of ileocolic intussusception.

The diagnosis of a typical case of HSP is a clinical one, and is often straightforward when the typical rash is present [2]. However, in at least $25 \%$ of cases, the rash appears after other manifestations, making early diagnosis challenging, like it occurred in the present patient, whose typical rash was preceded by recurrent painful knee joint swelling and abdominal pains. In a review of 100 patients by Saulsbury [7] the purpura was preceded by arthritis or gastrointestinal complications by up to 2 weeks in $43 \%$ of patients. The absence of the characteristic rash at onset may obscure diagnosis, while initial abdominal manifestations may suggest a surgical abdomen and warrant needless laparotomies [8].

Gastrointestinal manifestations are present in 50\% - 75\% of HSP cases [9]. Gastrointestinal symptoms usually manifest with abdominal pain which is characteristically colicky and localized to the periumbilical and epigastric regions 
[9] [10]. The pain worsens after meals, and may be associated with nausea and vomiting or transient ileus [9] [10]. Gastrointestinal symptoms may be due to inflammation, intestinal wall edema or hemorrhage, or more serious complications such, obstruction, infarction and intussusception [9]. Perforations, which are usually ileal, may occur spontaneously or be associated with intussusception [10].

Intussusception is rare in HSP and complicates $1 \%-5 \%$ of cases [7], it is however the commonest surgical complication of HSP [9] [10]. Bowel wall edema or submucosal bleeding are the typical identified lead points in intussusception associated with HSP [9]. The study of choice to diagnose intussusception is ultrasound, though barium enema may also be both diagnostic, and therapeutic.

It should be noted that no specific laboratory finding is diagnostic of HSP [2]. Investigations are required mostly to exclude other diagnosis as well as serious gastrointestinal or renal complications. Assessment of renal involvement with blood pressure, urinalysis, and serum creatinine is therefore necessary, while ultrasound is often used in the setting of gastrointestinal complaints to establish the presence of bowel wall edema or the rare occurrence of an associated intussusception. Other common, but nonspecific findings in HSP, include leukocytosis, thrombocytosis, mild anemia and an elevated erythrocyte sedimentation rate (ESR) [2]. These were all present in the presented case. Occult blood is frequently found in stool specimens in the absence of overt intestinal bleeding.

Autoantibody testing is generally not useful except to exclude other diseases, while serum IgA levels, though, often elevated, are not routinely measured [2]. Saulsbury [7] reported elevated IgA levels in $62 \%$ of the 71 patients in whom Immunoglobulins concentration were measured; 38\% had normal IgA levels, while none of the subjects had decreased IgA concentrations. Biopsies of skin or kidney are however unnecessary in typical HSP and should be reserved for atypical or severe cases [2]. They characteristically show IgA deposition in affected tissues.

This report also highlights the typically self-limiting nature of the disease in children. Treatment for mild disease is supportive, with emphasis on adequate hydration, nutrition, and pain control. The use of corticosteroids is controversial and reserved for patients with significant gastrointestinal involvement or other life-threatening manifestations. This was however unnecessary in the present case as she presented to us following resolution of joint disease and after laparotomy for intussusception. There was also no evidence of renal involvement.

Some reports have shown that Prednisolone reduces abdominal and joint pain in HSP [2]. It however does not alter overall prognosis nor prevent renal disease [2]. Rapid tapering of corticosteroids may also lead to a flare of HSP symptoms [2]. Intravenous immune globulin and plasma exchange have also been used in the setting of severe disease. Chronic HSP renal disease may require treatment with immunosuppressants such as azathioprine, cyclophosphamide, cyclosporine, and mycophenolate mofetil. 
Overall, the prognosis for childhood HSP is excellent [2], and most children experience an acute, self-limited course lasting on average of 4 weeks. The present patient's symptoms waxed and wane over a twelve-week period. About 15\% $60 \%$ of children with HSP experience one or more recurrences, typically within 4 months - 6 months of diagnosis. Calvo-Rio et al. [4] reported relapses in $32 \%$ of 417 reviewed cases, most of whom (75\%) were less than 20 years of age. The presence of joint and gastrointestinal symptoms at the time of diagnosis was identified as the best predictive index for relapse in the same study [4]. It has also been suggested that children with a severe initial disease course are at higher risk for relapse [2]. With each relapse, symptoms are usually milder than at presentation [2]. Our patient has however been symptom-free over a one year follow-up period, and her blood pressure as well as urinalysis have remained normal.

The long-term prognosis of HSP depends upon the severity of gastrointestinal or renal involvement. Acutely, serious gastrointestinal involvement such as intestinal perforation and intussusception impacts significantly on morbidity and mortality. Renal disease is, however, the major long-term complication, occurring in $1 \%-2 \%$ of children with HSP [2] [3], while end-stage renal disease develops in up to $8 \%$ of children with HSP nephritis. Renal disease can develop up to 6 months after diagnosis, but rarely occurs if initial urinalysis findings are normal [2].

\section{Conclusions}

This report highlights HSP as the commonest vasculitis in children. The diagnosis should be suspected in the presence, the typical palpable, non-thrombocytopenic purpura in children. The rash may however be preceded by other symptoms. A high index of suspicion for severe HSP-associated abdominal complications is therefore crucial to reduce morbidity from such complications.

It is recommended that children with HSP undergo serial monitoring of blood pressure and urinalyses for several months after diagnosis to monitor for the development of nephritis.

\section{Acknowledgements}

The authors would like to thank Abdurraheem NT of the Paediatric Surgery Unit, Federal Medical Centre Abeokuta who referred this case to us for evaluation. His continued interest and follow-up of the case is also appreciated.

\section{Conflicts of Interest}

The authors declare no conflicts of interest regarding the publication of this paper.

\section{References}

[1] Roberts, P.F., Waller, T.A., Brinker, T.M., Riffe, I.Z., Sayre, J.W. and Bratton, R.L. 
(2007) Henoch Schonlein Purpura: A Review Article. Southern Medical Journal, 100, 821-824. https://doi.org/10.1097/SMJ.0b013e3180f62d0f

[2] Sivaraman, V., Fels, E.C. and Ardoin, S.P. (2019) Vasculitis Syndromes: HenochSchonlein Purpura. In: Kliegman, R.M., St Geme, J.W., Blum, N.J., et al., Eds., Nelson Textbook of Paediatric (21 ed.), Elsevier, Philadelphia, 5400-5406.

[3] Saulsbury, F. (2002) Epidemiology of Henoch-Schonlein Purpura. Cleveland Clinic Journal of Medicine, 69, 87-89. https://doi.org/10.3949/ccjm.69.Suppl 2.SII87

[4] Calvo-Río, V., Hernández, J.L., Ortiz-Sanjuán, F., et al. (2016) Relapses in Patients with Henoch-Schönlein Purpura: Analysis of 417 Patients from a Single Center. Medicine, 95, e4217. https://doi.org/10.1097/MD.0000000000004217

[5] Hwang, H.H., Lim, I.S., Choi, B.-S. and Yi, D.Y. (2018) Analysis of Seasonal Tendencies in Pediatric Henoch-Schönlein Purpura and Comparison with Outbreak of Infectious Diseases. Medicine, 97, e12217. https://doi.org/10.1097/MD.0000000000012217

[6] Yang, Y.-H., Yu, H.-H. and Chiang, B.-L. (2014) The Diagnosis and Classification of Henoch-Schönlein Purpura: An Updated Review. Autoimmunity Reviews, 13, 355-358. https://doi.org/10.1016/j.autrev.2014.01.031

[7] Saulsbury, F.T. (1999) Henoch-Schönlein Purpura in Children. Report of $100 \mathrm{~Pa}$ tients and Review of the Literature. Medicine, 78, 395-409. https://doi.org/10.1097/00005792-199911000-00005

[8] Glasier, C., Siegel, M., McAlister, W. and Shackelford, G. (1981) Henoch-Schonlein Syndrome in Children: Gastrointestinal Manifestations. American Journal of Roentgenology, 136, 1081-1085. https://doi.org/10.2214/ajr.136.6.1081

[9] Lai, H.-C. (2010) Henoch-Schönlein Purpura with Intussusception: A Case Report. Pediatr Neonatol, 51, 65-67. https://doi.org/10.1016/S1875-9572(10)60013-7

[10] Ebert, E.C. (2008) Gastrointestinal Manifestations of Henoch-Schonlein Purpura. Digestive Diseases and Sciences, 53, 2011-2019. https://doi.org/10.1007/s10620-007-0147-0 